\title{
Direct Observation of Defects in Hexagonal Boron Nitride Monolayers
}

\author{
Peizhi Liu ${ }^{1}$, Junjie Guo ${ }^{1,2}$, Lei Liu $^{3}$, Wolfgang Windl ${ }^{4}$, Gong $\mathrm{Gu}^{3}$ and Gerd Duscher ${ }^{1,2}$ \\ 1. Department of Materials Science and Engineering, the University of Tennessee, Knoxville, TN 37996 , \\ United States \\ 2. Materials Science and Technology Division, Oak Ridge National Laboratory, Oak Ridge, TN 37831 , \\ United States \\ 3. Department of Electrical Engineering and Computer Science, the University of Tennessee, Knoxville, \\ TN 37996, United States \\ 4. Department of Materials Science and Engineering, Ohio State University, Columbus, OH 43210, \\ United States
}

Two-dimensional (2D) hexagonal boron nitride (h-BN) has a promising application in nanoelectronics. Here we want to explore how the electronic properties can be changed for optoelectronics applications through defect engineering. However, point defects, dislocations and grain boundaries of h-BN, which have been studied theoretically but not directly been seen in experiment, have not been as well understood as that in graphene, [1-3].

Here we resolved boron vacancies $\left(\mathrm{V}_{\mathrm{B}}\right)$, nitrogen vacancies $\left(\mathrm{V}_{\mathrm{N}}\right)$ nitrogen anti sites $\left(\mathrm{N}_{\mathrm{B}}\right)$, and dislocations in single layer $\mathrm{h}-\mathrm{BN}$ and its edges atom by atom. The state-of-the-art $5^{\text {th }}$ order aberration-corrected scanning transmission electron microscope (STEM, Nion UltraSTEM100) was used for atomic resolution imaging. The annular dark field (ADF) images from this dedicated STEM have the abilities of resolving light atoms such as boron, carbon, nitrogen, oxygen and so on at an acceleration energy of 60kV [4]. The monochromatic TEM (Libra200 MC), which have an energy resolution of $0.15 \mathrm{eV}$, was used for electron diffraction and electron energy-loss (EEL) spectra collection. Density functional theory (DFT) calculations and simulations were performed for comparison with experiments [5].

ADF images in Fig.1 (a), (b) and (c) show a $\mathrm{V}_{\mathrm{B}}$, a $\mathrm{V}_{\mathrm{N}}$ and a $\mathrm{N}_{\mathrm{B}}$ defects in h-BN monolayer respectively. Formation energies of all kinds of point defects in h-BN monolayer were calculated. Comparing the calculations with our observation, the h-BN monolayer manifested a nitrogen-rich tendency. More interestingly a new band shows up in the band gap of N-rich h-BN, shown in Fig. 1(d). As a result, h-BN changed from an insulator to a semiconductor by nitrogen doping. And the peak prior to the normal band gap peak in low loss EEL spectrum conformed to our calculation, shown in Fig. 1(e). The new band gap of $\sim 3.1 \mathrm{eV}$ corresponds to a visible light with the wavelength of $400 \mathrm{~nm}$. Fig. 1(f) is an ADF image of a dislocation in h-BN with a simulated atomic model. It shows that bonds around the dislocation core distorted into the $3^{\text {rd }}$ dimension. Fig. $1(\mathrm{~g})$ is an $\mathrm{AB}$ stacked h-BN double layer with a monolayer in the center. It displays that the h-BN edge can be ether an armchair shape or a zigzag shape. And a zigzag edge preferred to nitrogen terminations.

The results revealed direct evidences to the structures of defects in h-BN monolayer, and provide a method to tune the electronic and optical properties of h-BN monolayers by self-doping. 


\section{References:}

[1] Y. Liu, S. Bhowmick, and B.I. Yakobson, Nano Letters 11 (2011) p. 3113-3116.

[2] Y. Liu, X. Zou, and B.I. Yakobson, ACS Nano 6 (2012) p. 7053-7058.

[3] G.J. Slotman, and A. Fasolino, Journal of Physics: Condensed Matter 25 (2013) p. 045009

[4] O.L. Krivanek, M.F. Chisholm, V. Nicolosi, et al., Nature 464 (2010) p. 571-574.

[5] R. Mishra, O.D. Restrepo, A. Kumar, et al., Journal of Materials Science 47 (2012): p. $7482-7497$

[6] We acknowledge the Joint Institute for Advanced Materials, and the Division of Materials Sciences and Engineering, Office of Basic Energy Sciences of the U.S. Department of Energy for the use of TEM and STEM.
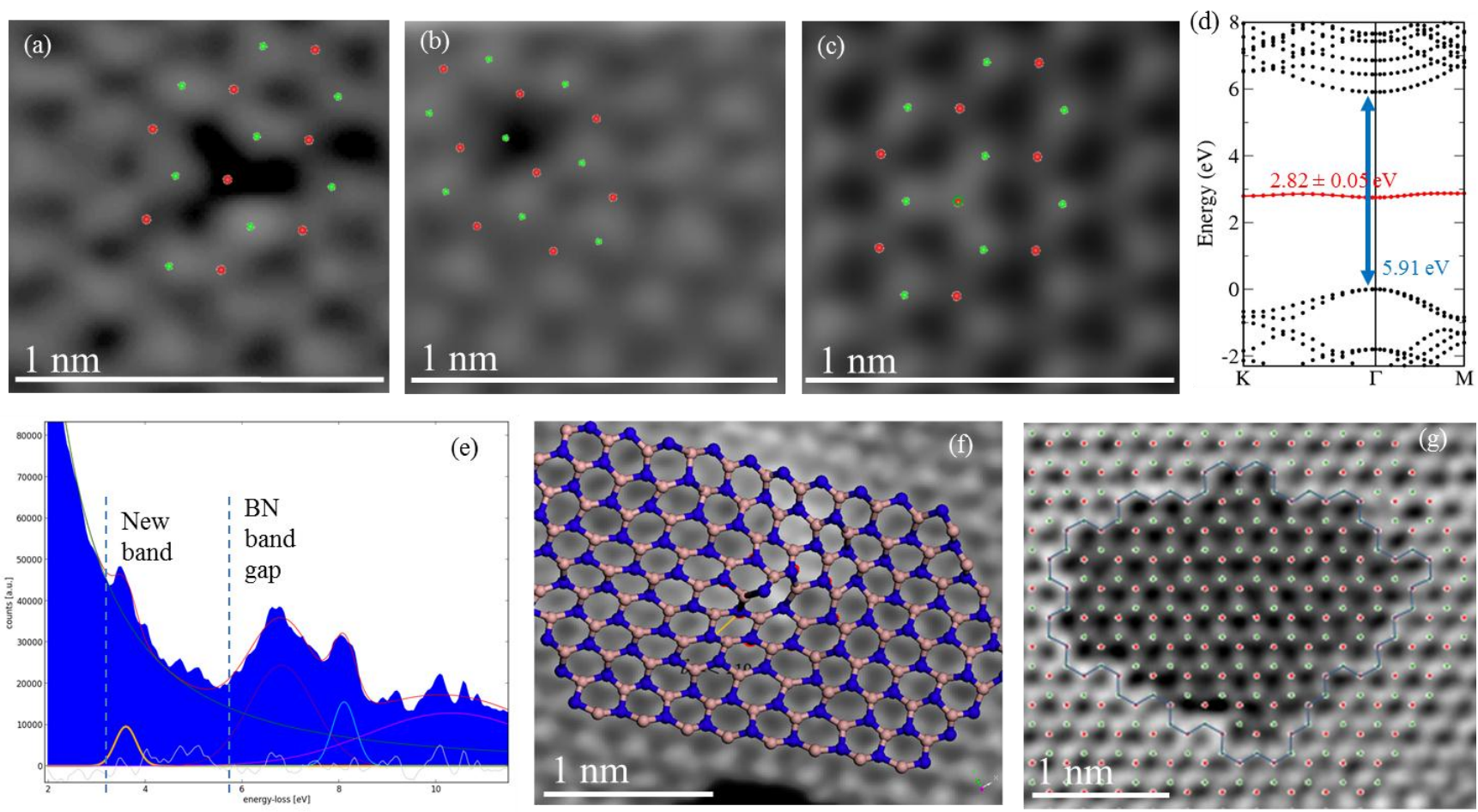

Figure 1. Defects in h-BN. (a), (b) (c) show a $V_{B}$, a $V_{N}$ and a $N_{B}$ respectively. (d) is the band structure of N-rich BN calculated with hybrid functionals. A $3 \times 3$ super cell was used in the calculation. (e) is a low loss EEL spectrum, which shows a new band prior to the normal band gap. (f) is a dislocation with the burgers vector $(1,1)$ and $(\mathrm{g})$ is a $\mathrm{AB}$ stacked $\mathrm{h}-\mathrm{BN}$ double layer with a monolayer in the center. Edges of the second layer were connected with solid lines. In h-BN atomic model, boron: red dot; nitrogen: green dot. 